where $A$ is the shaded area shown in Fig. 1, i.e.

$$
j_{+}(0)=\frac{1}{\pi T_{m f}} \tan ^{-1}\left\{\left(\frac{1-\gamma}{1+\gamma}\right)^{1 / 2}\right\} .
$$

For the process $A_{\gamma}$,

$$
j_{+}(0)=\frac{1}{\pi \tau_{0}(1-\gamma)} \tan ^{-1}\left\{\left(\frac{1-\gamma}{1+\gamma}\right)^{1 / 2}\right\} .
$$

As $\gamma$ approaches one, $j_{+}(0)$ becomes infinite. This will be true not only for zero but for all $x$. The implication is plain. The Fokker-Planck process is a degenerate process in which the one sided current density of the system is infinite. A Fokker-Planck model for the velocity motion of a colloid particle would describe an infinite number of changes of direction of the particle per unit time. Such a model used to describe voltage fluctuations would imply an infinite number of polarity reversals per second. Since a process $A \gamma$ will afford the same correlation function and equilibrium distribution, and finite polarity reversal frequency, it is suggested that such a model may better describe noise, and that the number of zero crossings be regarded as an independent macroscopic physical quantity on an equal footing with $\tau_{0}, E_{0}$.

The author thanks Dr. Franz Stumpers of Phillips Eindhoven and Dr. Edwin Akutowicz for their interest and encouragement.

\title{
EVALUATION OF CONSTANTS IN CONFORMAL REPRESENTATION*
}

By SAMUEL I. PLOTNICK ANd THOMAS C. BENTON (Pennsylvania State University)

In using the Schwarz-Christoffel transformation [1],

$$
d z=K \prod_{i=1}^{n}\left(\zeta-\zeta_{i}\right)^{\left(\alpha_{i} / \pi\right)-1} d \zeta=K f(\zeta) d \zeta
$$

whereby the upper half $\zeta$-plane is mapped into a simple connected polygon, the evaluation of the unknown constant $K$ (if complex $K=c e^{i \lambda}, c, \lambda$ real), is oftentimes tedious. We shall show a simple method of evaluating the unknown constant $K$ by examples, proving first a

TheоRem: By the Schwarz-Christoffel transformation if $\zeta_{i}$ in the $\zeta$-plane corresponds to two points $P_{i}, Q_{i}$ in the z-plane and $\zeta=\zeta_{i}$ is a simple pole of $f(\zeta)$, then

$$
K=\frac{\operatorname{dist}\left(P_{i}, Q_{i}\right)}{\pi i R\left(\zeta=\zeta_{i}\right)}
$$

$R$, denoting residue and dist $\left(P_{i}, Q_{i}\right)$, denoting the distance between the two points $P_{i}$ and $Q_{i}$.

${ }^{*}$ Received May 8, 1953. 
Proof: Since

$$
\begin{aligned}
d z & =K f(\zeta) d \zeta \\
\int_{P_{i}}^{0_{i}} d z & =\operatorname{dist}\left(P_{i}, Q_{i}\right) \\
& =\operatorname{Lim}_{\delta \rightarrow 0} \int_{0}^{\pi} K f\left(\zeta_{i}+\delta e^{i \theta}\right) i \delta e^{i \theta} d \theta \\
& =K \operatorname{Lim}_{\delta \rightarrow 0} \int_{0}^{\pi} f\left(\zeta_{i}+\delta e^{i \theta}\right) i \delta e^{i \theta} d \theta,
\end{aligned}
$$

where $\zeta-\zeta_{i}=\delta e^{i \theta}$.

Since $f$ has a simple pole at $\zeta_{i}$ the Laurent expansion [2], is

$$
f(\zeta)=\frac{R}{\zeta-\zeta_{i}}+g(\zeta)
$$

with $g(\zeta)$ analytic near $\zeta=\zeta_{i}$. Now,

$$
f\left(\zeta_{i}+\delta e^{i \theta}\right)=\frac{R}{\delta e^{i \theta}}+g\left(\zeta_{i}+\delta e^{i \theta}\right) .
$$

Therefore,

$$
\begin{aligned}
\operatorname{Lim}_{\delta \rightarrow 0} \int_{0}^{\pi} f\left(\zeta_{i}+\delta e^{i \theta}\right) i \delta e^{i \theta} d \theta & =\operatorname{Lim}_{\delta \rightarrow 0}\left[R i \int_{0}^{\pi} d \theta+i \delta \int_{0}^{\pi} \sum_{k=0}^{\infty} c_{k} \delta^{k} e^{(k+1) i \theta} d \theta\right] \\
& =i \pi R
\end{aligned}
$$

whence

$$
K=\frac{\operatorname{dist}\left(P_{i}, Q_{i}\right)}{\pi i R\left(\zeta=\zeta_{i}\right)}
$$

Consider the transformation described by Milne-Thomson [3], whereby we map the infinite strip on the upper half $\zeta$-plane. The Schwarz-Christoffel transformation gives

$$
\frac{d z}{d \zeta}=K \zeta^{-1} \quad \text { or } \quad z=K \int \frac{d \zeta}{\zeta}+L .
$$

$L=0$ for $z=0$ corresponds to $\zeta=1$.

By the theorem,

$$
K=\frac{\operatorname{dist}\left(P_{i}, Q_{i}\right)}{\pi i R(\zeta=1)}=\frac{a i}{\pi i(1)}=\frac{a}{\pi}
$$

In this case comparison of real and imaginary parts with infinities is avoided.

\section{References}

1. Zeev-Nehari, Conformal mapping, McGraw Hill, 1952, pp. $189 \mathrm{ff}$.

2. E. C. Titchmarsh, The theory of functions, 2nd Ed., Oxford University Press, 1950, p. 91.

3. L. M. Milne-Thomson, Theoretical hydrodynamics, 2nd. Ed., The Macmillan Company, London, 1950, p. 275. 